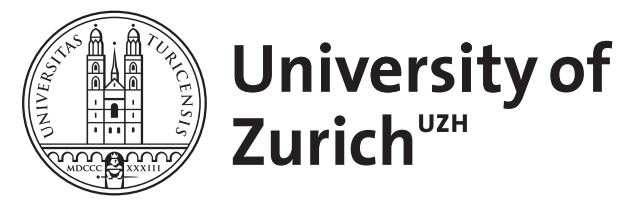

Zurich Open Repository and Archive

University of Zurich

University Library

Strickhofstrasse 39

CH-8057 Zurich

www.zora.uzh.ch

Year: 2011

Clinical value of a combined multi-phase contrast enhanced DOPA-PET/CT in neuroendocrine tumours with emphasis on the diagnostic CT component

Veit-Haibach, P ; Schiesser, M ; Soyka, J ; Strobel, K ; Schaefer, N G ; Hesselmann, R ; Clavien, P A ; Hany, T F

\begin{abstract}
DOPA cePET/CT was significantly more accurate than DOPA-PET alone and ceCT alone. The CT component itself had a diagnostic impact in a small percentage but contributed to the therapeutic strategies in selected patients.
\end{abstract}

DOI: https://doi.org/10.1007/s00330-010-1930-4

Posted at the Zurich Open Repository and Archive, University of Zurich ZORA URL: https://doi.org/10.5167/uzh-45969

Journal Article

Published Version

Originally published at:

Veit-Haibach, P; Schiesser, M; Soyka, J; Strobel, K; Schaefer, N G; Hesselmann, R; Clavien, P A; Hany, T F (2011). Clinical value of a combined multi-phase contrast enhanced DOPA-PET/CT in neuroendocrine tumours with emphasis on the diagnostic CT component. European Radiology, 21(2):256-264.

DOI: https://doi.org/10.1007/s00330-010-1930-4 


\title{
Clinical value of a combined multi-phase contrast enhanced DOPA-PET/CT in neuroendocrine tumours with emphasis on the diagnostic CT component
}

\author{
Patrick Veit-Haibach • Marc Schiesser • Jan Soyka • \\ Klaus Strobel • Niklaus G. Schaefer • Rolf Hesselmann • \\ P.-A. Clavien - Thomas F. Hany
}

Received: 10 May 2010 / Revised: 22 July 2010 /Accepted: 28 July 2010 /Published online: 15 August 2010

(C) European Society of Radiology 2010

\begin{abstract}
Objective To assess the clinical value of multi-phase, contrast-enhanced DOPA-PET/CT with emphasis on the diagnostic $\mathrm{CT}$ component in patients with neuroendocrine tumours (NET).

Methods Sixty-five patients with NET underwent DOPAcePET/CT. The DOPA-PET, multi-phase CT and combined DOPA cePET/CT data were evaluated and diagnostic accuracies compared. The value of ceCT in DOPA cePET/ $\mathrm{CT}$ concerning lesion detection and therapeutic impact was evaluated. Sensitivities, specificities and accuracies were calculated. Histopathology and clinical follow-up served as the standard of reference. Differences were tested for statistical significance by McNemar's test.

Results In 40 patients metastatic and/or primary tumour lesions were detected. Lesion-based analysis for the DOPA-
\end{abstract}

P. Veit-Haibach $(\bowtie) \cdot J$. Soyka $\cdot$ K. Strobel $\cdot$ N. G. Schaefer $\cdot$

R. Hesselmann - T. F. Hany

Department of Medical Radiology, Division of Nuclear Medicine,

University Hospital Zurich,

Rämistrasse 100,

8091 Zuerich, Switzerland

e-mail: patrick.veit@gmx.de

M. Schiesser · P.-A. Clavien

Department of Surgery, University Hospital Zurich,

Zurich, Switzerland

N. G. Schaefer

Department of Oncology, University Hospital Zurich,

Zurich, Switzerland

Present Address:

P. Veit-Haibach

Department of Radiology and Nuclear Medicine,

Lucerne Cantonal Hospital,

Lucerne, Switzerland
PET showed sensitivity, specificity and accuracy of $66 \%$, $100 \%$ and $67 \%$, for the ceCT data $85 \%, 71 \%$ and $85 \%$, and for the combined DOPA cePET/CT data $97 \%, 71 \%$ and $96 \%$. DOPA cePET/CT was significantly more accurate compared with dual-phase CT $(p<0.05)$ and PET alone $(p<$ 0.05 ). Additional lesion detection was based on ceCT in 12 patients; three patients underwent significant therapeutic changes based on the ceCT findings.

Conclusion DOPA cePET/CT was significantly more accurate than DOPA-PET alone and ceCT alone. The CT component itself had a diagnostic impact in a small percentage but contributed to the therapeutic strategies in selected patients.

Keywords DOPA PET/CT · Multi-phase CT .

Therapeutic impact $\cdot$ Neuroendocrine tumors

\section{Introduction}

Neuroendocrine tumours (NET) are a heterogeneous group of neoplasms with an incidence of 100,000 per year in the U.S. and thus, represent a larger group of cancer patients than pancreatic cancer, gastric cancer, oesophageal cancer or hepatobiliary cancer [1-4]. The course of the disease depends on time of diagnosis, clinical staging, histopathology, grading and consecutive therapy. The time point of diagnosis is usually late after the disease onset, because a significant number of patients are asymptomatic for a long time [2] . Therefore, accurate and early diagnosis are crucial for the prognosis of these patients. Generally, staging includes morphological imaging techniques (computed tomography (CT) and/or magnetic resonance imaging (MRI)) to provide anatomical information about local- 
isation of the primary tumour as well as number and localisation of metastases. In CT, an arterial contrast phase as well as a portal-venous phase (or late venous phase) is needed for localisation and characterisation of NET. In addition, standard somatostatin receptor scintigraphy (SRS) is generally used to localise and characterise the NET. However, a significant rate of false negative results with sensitivities ranging between $50 \%$ and $78 \%$, have been reported [2, 5-9]. Positron emission tomography (PET) using the catecholamine precursor 6-(fluoride-18) fluorodopa (F18-DOPA) has been proposed as an alternative imaging option for NET and has already been shown to have a significant therapeutic impact in selected patient populations with NETs [7, 10-12]. Combined FDG-PET/ CT is already known to overcome the drawbacks of CT alone and PET alone in a variety of tumours [13-16]. So far, there has been no broad experience concerning the diagnostic accuracy of a combined, triple-phase contrastenhanced DOPA-PET/CT (DOPA cePET/CT) in the NETs available and the particular additional value concerning diagnosis and therapy of NETs of the ceCT component of such a combined protocol has not been evaluated.

Thus, the aim of our study was to prospectively evaluate: 1. the overall diagnostic accuracy of DOPA cePET/CT, multiphase CT (ceCT) and PET only in a lesion-based, region-based and patient-based analyses in a heterogeneous patient population with NET and 2. to evaluate the incremental diagnostic and therapeutic value of ceCT in such a combined protocol.

\section{Materials and methods}

\section{Patients}

Sixty-five consecutive patients (mean age: 56 years, range: $16-80$ years, 35 female, 30 male) were prospectively included between November 2006 and January 2009 in this study. The median follow-up time was 12 months (range 2-26 months, mean follow-up time: 13 months). Patients with suspicion of newly occurred neuroendocrine tumours based on clinical symptoms, laboratory findings or previous imaging (30 patients) and patients already known to have a neuroendocrine tumour and who were operated on or treated with systemic therapy (35 patients) were included in this study. Patients were included with no further selection based on the time point of their referral. Patients with severe claustrophobia and pregnant women were excluded. The study was performed in accordance with the regulations of the local institutional review board and ethics committee. Written informed consent was obtained from all patients before the DOPA cePET/CT-study.

\section{PET/CT imaging}

All data were acquired on a combined PET/CT in-line system (Discovery VCT, GE Healthcare, Milwaukee, WI, USA). This dedicated system integrates full-ring PET system with multislice helical 64-slice CT and permits the acquisition of co-registered CT and PET images in one imaging procedure.

$\mathrm{PET} / \mathrm{CT}$ imaging was started $45 \mathrm{~min}$ after the injection of a standard dose of 200-220 MBq F18-DOPA. In addition, $500 \mathrm{ml}$ of tap water was given to the patient $10 \mathrm{~min}$ before imaging. All patients initially underwent CT without intravenous contrast medium. This was acquired with the following parameters: $80 \mathrm{~mA}, 140 \mathrm{kV}$, 0.5 -second tube rotation, $4.25-\mathrm{mm}$ section thickness. Imaging extendedfrom the vertex to the upper thighs. Directly after this unenhanced CT data had been acquired, the emission PET Data acquisition was initiated with an acquisition time of 2 min per bed position. Then, intravenous contrast medium was administered by injecting a total dose of 90-120 ml Ultravist 300 (Bayer Schering Pharma) with a flow of $4 \mathrm{ml} / \mathrm{sec}$ for an arterially contrast-enhanced CT for the abdomen and/or thorax and abdomen.

After a delay of 70 seconds, another ceCT $(120 \mathrm{kV}$, dose-modulated tube current up to $700 \mathrm{~mA}, 2.5 \mathrm{~mm}$, pitch $1.375: 1,13.75 \mathrm{~mm} /$ rotation speed) was applied to ensure a portal venous phase. The initially acquired non-enhanced low-dose CT data (3.75-mm slices) were used for attenuation correction, and images were reconstructed by using a standard fully 3 D-iterative algorithm (ordered subset expectation maximisation, OSEM). For image coregistration and analysis, all reconstructed images were then transferred to a commercially available workstation (Advantage Workstation, 4.4, GE Healthcare, Milwaukee, WI, USA), where image data can be evaluated in all three planes as single procedures (multi-phase CT-only, PETonly) and in a co-registered mode (co-registered PET/CT). Thus, all tomographic imaging techniques (multi-phase CT only, PET only and DOPA cePET/CT) were derived from the same imaging procedure.

Imaging analysis

The participating readers were informed about the patientspecific clinical background (e.g. suspected diagnosis), but were blinded to the results of the other imaging procedures and the results of the clinical investigation (e.g. endoscopy, urine analysis). PET only and DOPA cePET/CT images were evaluated qualitatively for areas of focally increased DOPA metabolism above the surrounding tissue level. No quantitative assessment (e.g. Standard Uptake Value) was used for further lesion characterisation. On combined 
DOPA-cePET/CT, lesion detection (primary tumour, lymph node metastases, distant metastases) was based on the detection of soft tissue masses with arterial and/or venous contrast enhancement in conjunction with increased F18DOPA activity. Lymph nodes on combined DOPA cePET/ CT imaging were assessed for metastatic spread based on increased F18-DOPA activity and independent of their size.

On PET only imaging, lesion detection and characterisation were derived from the same criteria as on DOPA cePET/CT-imaging, however, without the evaluation of the CT component.

On multi-phase CT images (ceCT), detection of soft tissue masses with arterial and/or venous contrast enhancement characterised malignancy. Lymph node assessment on CT only imaging was based on lesion size (threshold $1-\mathrm{cm}$ short-axis diameter) [17]. Central necrosis was defined as a sign of malignancy as well, independent of lymph node size. A fatty hilum as well as calcifications were used as benign criteria on ceCT.

All evaluations were performed on a lesion-based analysis, a region-based analysis and a patient-based analysis.

Standard of reference (including SRS imaging)

In all patients, histopathological evaluation (surgical specimen, biopsy) as well as the clinical course containing all available clinical data (physical examination, further imaging including SRS imaging, laboratory findings) served as the standard of reference. In 36 patients histopathological verification of at least one lesion was available based on surgical procedures after DOPA imaging (17 patients), the remaining patients had biopsy or surgical procedures before DOPA-PET/CT imaging. The remaining four patients (of 40 patients with lesions, see "Results") did not receive histopathological evaluation for various reasons (e.g. typical laboratory findings and concomitant typical imaging findings, typical imaging findings but patient refused to have the biopsy, typical imaging findings but patient was not operated on because of good clinical condition and age, previous neuroendocrine tumour with recurrent rise of tumour markers and concomitant imaging findings). In the remaining patient population (no pathological findings in DOPA-PET/CT, see "Results" below), the clinical course containing the above-mentioned criteria and eight biopsy procedures served as the standard of reference. Mean follow-up time was 13 months (range 2-26 months).

In 49 patients, whole-body planar SRS imaging containing anterior and posterior views was performed $5 \mathrm{~h}$ and 24-30 $\mathrm{h}$ after the injection of 150-200 MBq of In-111Octreotide. Scintigraphy was performed on different systems with a medium energy collimator. Patients underwent SPECT imaging in the area of interest in the event of suspicious findings on planar imaging (45 patients). Four patients received no additional SRS imaging because the diagnosis could be clearly made on planar imaging and the nuclear medicine physician responsible decided to skip the additional SPECT procedure.

\section{Statistical analysis}

The primary endpoint of the study was the correct lesion detection and characterisation using CT only and PET only in comparison to combined DOPA-cePET/CT. Differences in the assessment of lesion detection between the different imaging procedures were tested for significance by McNemar's test (exact). A p value $<0.05$ was considered significant. Confidence intervals for the accuracy were calculated with a two-sided single proportion test. Sensitivities, specificities and accuracies (with exact 95\% confidence intervals) for all techniques were determined using histology and/or clinical follow-up as the standard of reference. Statistical analyses were performed with Microsoft Excel and MedCalc Software (Version 11.0.1.0 Broekstraat 52, 9030 Mariakerke, Belgium).

\section{Results}

\section{Patients}

All of the 65 patients evaluated had sufficient follow-up. Forty-nine patients had SRS imaging. In 20 patients, no suspicious findings were found with any of the imaging techniques under evaluation. In all 20 patients, no other imaging technique detected a neuroendocrine tumour during the clinical follow-up. In the remaining 45 patients, 227 neuroendocrine tumours/lesions were detected. The main body compartments affected were: liver (124 lesions), other intraperitoneal/retroperitoneal abdominal lesion (42 lesions), skeleton (27 lesions) thorax/neck (26 lesions) and the small bowel (eight lesions). In 36 patients, histopathological verification was available. Overall, $11(17 \%)$ patients had (or had previously during clinical work-up) a neuroendocrine tumour (carcinoid) of the pancreas, 10 $(15 \%)$ patients had a tumour (carcinoid/neuroendocrine carcinoma) of the small bowel, 5 (8\%) had pheochromocytomas, $4(6 \%)$ had medullary thyroid carcinomas, $2(4 \%)$ had other paragangliomas, in $2(4 \%)$ patients we detected only carcinoid metastases to the liver without a detectable primary, one patient had a neuroendocrine carcinoma of the lung and one patient had a neuroendocrine tumour of the stomach. Overall, 23 patients had metastatic lesions of different NETs to the liver. In eight patients, the primary tumour (6 small bowel lesions, two pancreatic lesions) were detected in the DOPA-cePET/CT, 5 of those primary 
tumours were previously unknown (Fig. 1). In one patient (with primary NET of the pancreatic head), an additional breast cancer with slight DOPA activity was detected and confirmed by mammography and histopathology.

Overall diagnostic accuracy

Overall, 227 lesions were detected. Of these, 158, 193 and 218 lesions were correctly evaluated (true positive and true negative) by DOPA-PET, ceCT and combined cePET/CT. Seventy-four lesions, 34 lesions and nine lesions were evaluated incorrectly (false negative and false positive) on DOPA-PET, ceCT and combined DOPA-cePET/CT.

Lesion-, region- and patient-based analyses for DOPA PET only, ceCT and combined DOPA-cePET/CT are shown in Tables 1 and 2. Overall, the DOPA-PET only shows moderate sensitivity and accuracy. On the other hand, it provides an optimal specificity.

Conversely, ceCT had higher sensitivity but lower specificity than DOPA-PET. These results are mainly based on two false positive lesions (two small liver lesions with arterial enhancement) which were falsely evaluated as metastases. In one patient the combined DOPA-cePET/CT was falsely negative - a NET of the pancreatic head was not detectable and was only visible on MRI (Fig. 2). Overall ceCT was found to be statistically more accurate than DOPA PET only ( $p<0.05$, lesion-based analysis) while combined DOPA-cePET/CT was statistically more accurate than ceCT $(p<0.05)$ and DOPA PET only $(p<0.05)$.

Incremental diagnostic and therapeutic value of the $\mathrm{CT}$ component

DOPA-cePET/CT was diagnostically superior to PET only based on the multi-phase CT component in 12 patients. One of these patients was post-liver transplant, and the ce-CT component detected DOPA-negative liver and lung metastases. However, there was no change in therapy because the patient showed stable disease. In one patient postsandostatin-therapy, the ce-CT component detected multiple liver metastases. Also here, no change in therapy was induced.
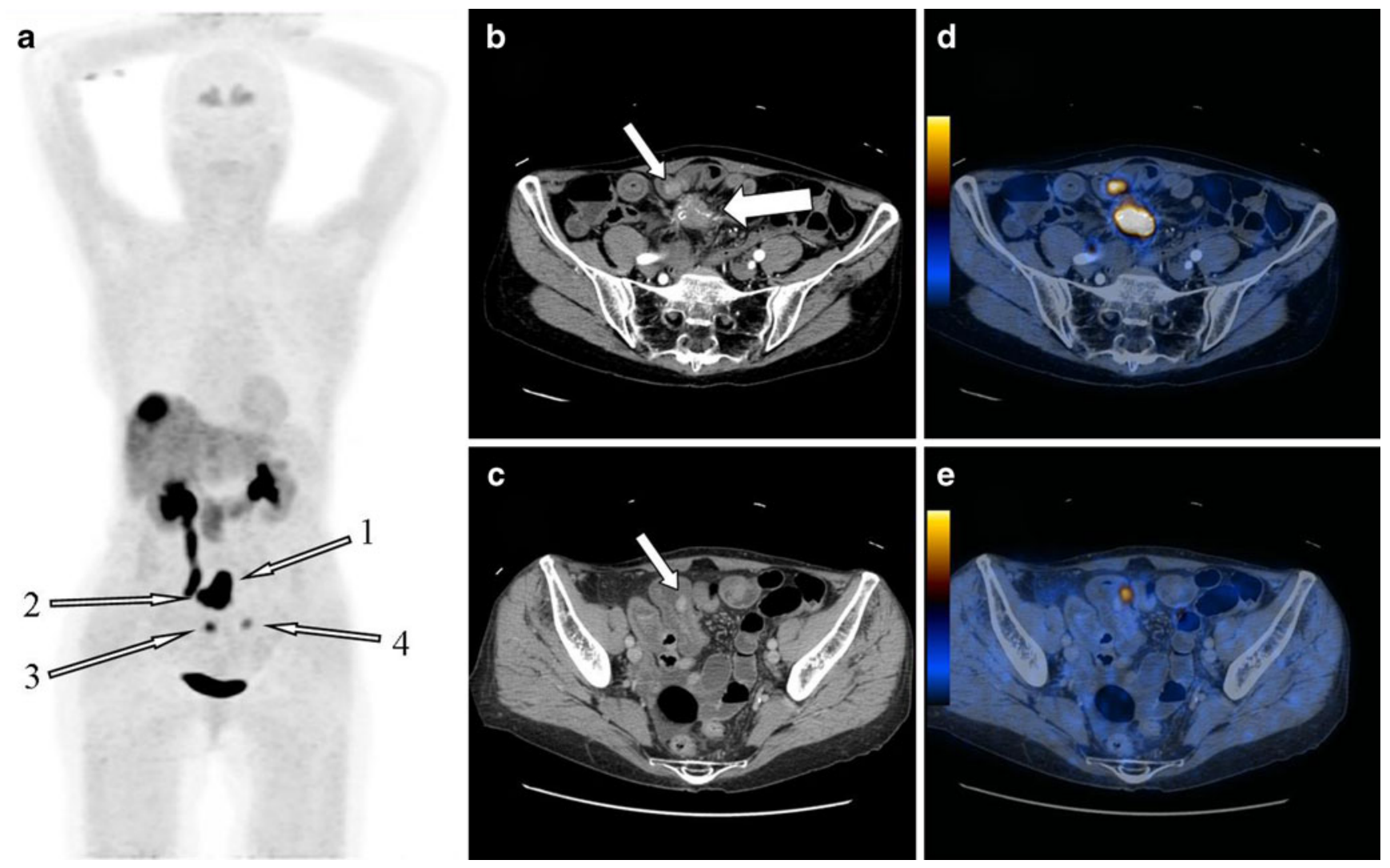

Fig. 1 a MIP, $\mathbf{b}$ and $\mathbf{d}$ axial contrast-enhanced CT, $\mathbf{c}$ and e combined DOPA-cePET/CT. Seventy-five-year-old woman with known liver metastases of a well-differentiated neuroendocrine carcinoma. A mesenteric lymph node was known from serial CTs and SRS imaging (a arrow Nr. 1, b big white arrow). A primary tumour had so far not been detected on previous morphological imaging and SRS imaging. On combined DOPA-cePET/CT, three primary tumours were detected within the jejunum. One primary tumour was detected directly ventrally within the jejunal wall (a arrow $\mathrm{Nr}$. 2, b small white arrow, c corresponding DOPA-cePET/CT), another was detected more distally in the jejunal wall (a arrow Nr. 3, d white arrow, e corresponding DOPA -cePET/CT) and the last primary was again detected more distally in the jejunum (a arrow Nr. 4, no axial slices shown) 
Table 1 shows the lesion-based and patient-based sensitivities, specificities, accuracies and corresponding $95 \%$ confidence intervals of the DOPA-PET, multi-phase CT and combined DOPA-cePET/CT evaluation

\begin{tabular}{lll}
\hline DOPA-PET & Lesion-based analysis & Patient-based analysis \\
Sensitivity $(95 \%-C I)$ & $66 \%(60-72 \%)$ & $82 \%(66-92 \%)$ \\
Specificity $(95 \%-C I)$ & $100 \%(63-100 \%)$ & $100 \%(87-100 \%)$ \\
Accuracy $(95 \%-C I)$ & $67 \%(61-73 \%)$ & $89 \%(79-96 \%)$ \\
Mutli-phase CT & & \\
Sensitivity $(95 \%-C I)$ & $85 \%(80-90 \%)$ & $93 \%(80-98 \%)$ \\
Specificity $(95 \%-C I)$ & $71 \%(29-96 \%)$ & $100 \%(86-100 \%)$ \\
Accuracy $(95 \%-C I)$ & $85 \%(80-89 \%)$ & $95 \%(87-99 \%)$ \\
Combined DOPA-cePET/CT & & $98 \%(87-100 \%)$ \\
Sensitivity $(95 \%-C I)$ & $97 \%(94-99 \%)$ & $100 \%(86-100 \%)$ \\
Specificity $(95 \%-C I)$ & $71 \%(29-96 \%)$ & $98 \%(92-100 \%)$ \\
Accuracy $(95 \%-C I)$ & $96 \%(93-98 \%)$ & \\
\hline
\end{tabular}

In another patient, a DOPA-negative, largely cystic pancreatic tumour was detected. The patient was operated based on the CT findings and an adeno-carcinoma was identified by histopathological evaluation. In one patient, the ce-CT detected very small recurrent liver metastases 7 years after surgery of a carcinoid tumour. Imaging findings did not lead to a therapy change, because the lesions were very small. In one patient a small neuroendocrine tumour of the jejunum was DOPA-negative and was only detected by its arterial enhancement on ce-CT (Fig. 3). The patients underwent surgery of the primary tumour. In one patient, the CT component detected the primary tumour, concurrent lymph nodes and liver metastases of a known pancreatic NET. In another patient, the CT component identified the DOPAnegative liver and bone metastases of an unknown, as yet undiscovered primary NET. In both patients, previous systemic therapy was not changed because the imaging confirmed the suspected sites of disease. Lastly, in one patient, small (DOPA-negative) liver metastases were suggested based on their arterial enhancement. The patient underwent surgery and histopathology revealed small adenomas with ectatic feeding vessels. In four patients, the ceCT component detected just additional metastases compared with the already detected DOPA-positive metastases (liver metastases, lymph node metastases, bone metastases). These additional findings led to a therapy change from previously intended surgical therapy to a systemic therapy approach in one patient.

\section{Discussion}

Comparison with conventional imaging

Several studies already investigated the usefulness of DOPA-PET and PET/CT concerning the diagnostic accura-

Table 2 shows the region-based sensitivities, specificities, accuracies and corresponding $95 \%$ confidence intervals of the DOPA-PET, multiphase CT and combined DOPA-cePET/CT evaluation

\begin{tabular}{|c|c|c|c|c|c|}
\hline DOPA-PET & Liver & Abdominal lesions & Thorax/neck lesions & Small bowel & Bone \\
\hline Sensitivity $(95 \% \mathrm{CI})$ & $63 \%(54-71 \%)$ & $77 \%(6189 \%)$ & $64 \%(41-83 \%)$ & $88 \%(47-100 \%)$ & $59 \%(38-78 \%)$ \\
\hline Specificity $(95 \% \mathrm{CI})$ & $100 \%(16-100 \%)$ & $100 \%(16-100 \%)$ & $100 \%(40-100 \%)$ & $\mathrm{n} / \mathrm{a}$ & $\mathrm{n} / \mathrm{a}$ \\
\hline Accuracy $(95 \% \mathrm{CI})$ & $63 \%(54-72 \%)$ & $78 \%(62-89 \%)$ & $69 \%(48-86 \%)$ & $88 \%(47-100 \%)$ & $59 \%(39-78 \%)$ \\
\hline \multicolumn{6}{|l|}{ Multi-phase CT } \\
\hline Sensitivity $(95 \% \mathrm{CI})$ & $90 \%(83-95 \%)$ & $95 \%(83-99 \%)$ & $73 \%(50-89 \%)$ & $50 \%(16-84 \%)$ & $70 \%(50-86 \%)$ \\
\hline Specificity $(95 \% \mathrm{CI})$ & $0 \%(0-84 \%)$ & $100 \%(3-100 \%)$ & $100 \%(40-100 \%)$ & $\mathrm{n} / \mathrm{a}$ & $\mathrm{n} / \mathrm{a}$ \\
\hline Accuracy $(95 \% \mathrm{CI})$ & $89 \%(82-94 \%)$ & $95 \%(83-99 \%)$ & $77 \%(56-91 \%)$ & $50 \%(16-84 \%)$ & $70 \%(50-86 \%)$ \\
\hline \multicolumn{6}{|c|}{ Combined DOPA-cePET/CT } \\
\hline Sensitivity $(95 \% \mathrm{CI})$ & $96 \%(91-99 \%)$ & $98 \%(87-100 \%)$ & $100 \%(85-100 \%)$ & $100 \%(63-100 \%)$ & $96 \%(81-99 \%)$ \\
\hline Specificity $(95 \% \mathrm{CI})$ & $0 \%(0-84 \%)$ & $100 \%(3-100 \%)$ & $100 \%(40-100 \%)$ & $\mathrm{n} / \mathrm{a}$ & $\mathrm{n} / \mathrm{a}$ \\
\hline Accuracy $(95 \% \mathrm{CI})$ & $94 \%(89-98 \%)$ & $98 \%(87-100 \%)$ & $100 \%(87-100 \%)$ & $100 \%(63-100 \%)$ & $96 \%(81-100 \%)$ \\
\hline
\end{tabular}

Liver only liver lesions were counted and evaluated, Abdominal Lesions other abdominal (other than liver or small bowel) soft tissue/organ lesions and lymph nodes were counted and evaluated, Thorax/Neck Lesions soft tissue/organ (pulmonary lesions and lymph nodes were counted and evaluated, Small Bowel only lesions of the small bowel were counted and evaluated, Bone bone lesions within the field-of-view (FOV head to the upper thighs) were counted and evaluated, $n / a$ not applicable (no true negatives, no false positives in this category) 

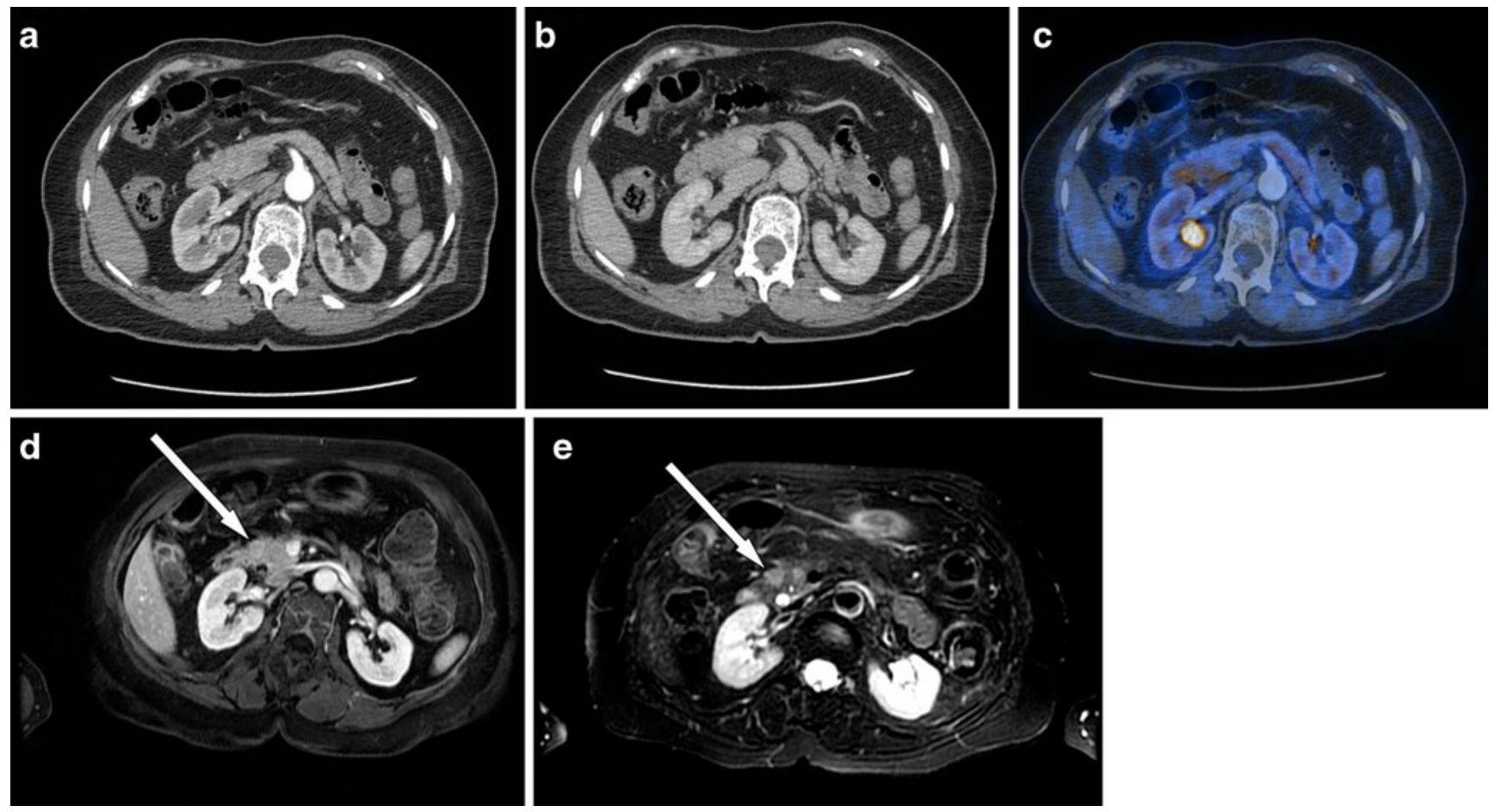

Fig. 2 a contrast-enhanced (arterial phase) axial CT, b contrastenhanced (venous phase) axial CT, c combined DOPA-cePET/CT, d corresponding T1-weighted, post-gadolinium, fat-saturated, axial MRI, e T2-weighted, fat-saturated, axial MRI. Seventy-two-year-old woman with clinical signs of a neuroendocrine tumour and follow-up after

detected, neither on dual-phase CT (a and $\mathbf{b}$ ), nor on combined DOPAcePET/CT (c). Only the corresponding MRI showed multifocal lesions within the pancreatic head (see arrows in $\mathbf{d}$ and e). Lesions were surgically removed and confirmed as well-differentiated, multifocal, lung tumour surgery. A suspect lesion of the pancreas could not be

Fig. 3 a contrast-enhanced (arterial phase) axial CT, b corresponding DOPA-PET/CT, c corresponding MIP image. Sixty-three-year-old male patient with clinical signs of a carcinoid. The arterial phase of the contrast-enhanced DOPAcePET/CT showed a focal, arterially enhancing lesion of the jejunum (white arrow, a), however, without DOPA-activity (b). Corresponding MIP image (c) also showed no pathological DOPA activity in this patient. The tumour was diagnosed solely on the ceCT component
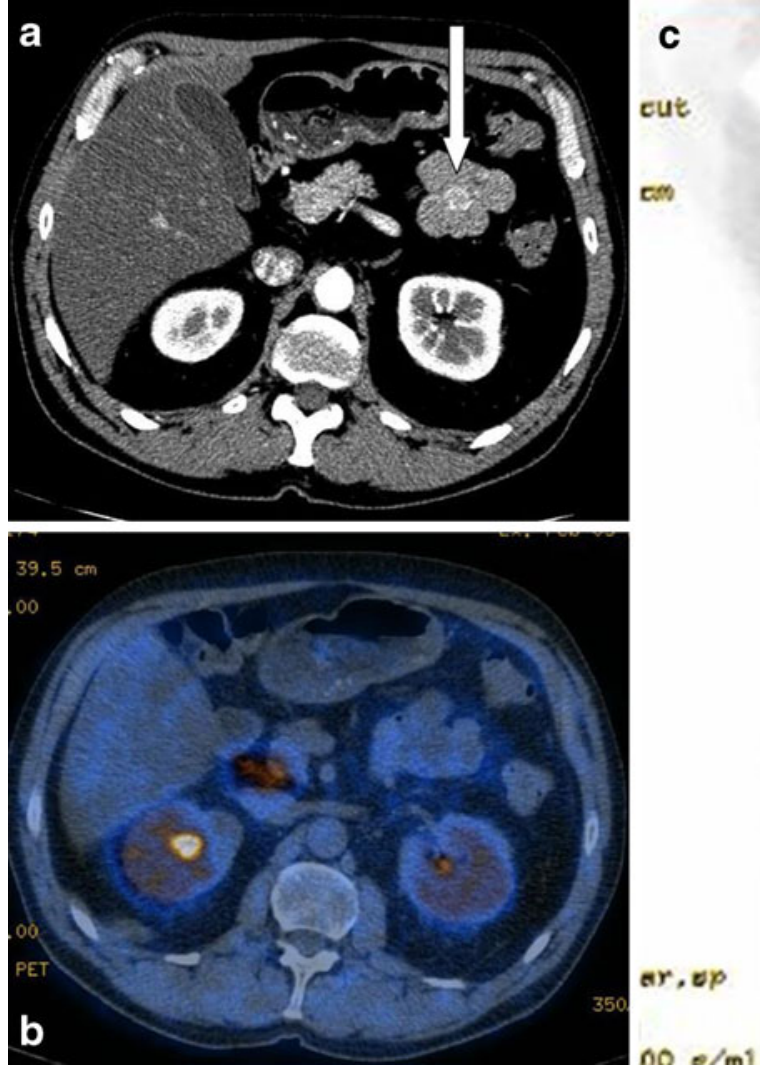

129 Dot 
cy in a variety of NETs and its subtypes [6, 18-23]. The diagnostic superiority of DOPA-PET over conventional SRS imaging has been demonstrated in several studies [7, 24, 25]. While Hoegerle et al. reported in a small patient population an overall sensitivity of $65 \%$ for DOPA-PET in a lesion-based analysis, Koopmans and co-workers found lesion-based sensitivities ranging between $95 \%$ and $100 \%$ $[6,7]$. Moreover, DOPA-imaging proved to be useful in patients in whom SRS imaging was negative [26].

Overall diagnostic value of DOPA-PET/CT and comparisons with other tracers

DOPA-PET and PET/CT already demonstrated high diagnostic values when evaluating heterogeneous patient populations and several subtypes of NETs $[6,7,25]$. Similar results concerning region-based analysis were found e.g. by Becherer et al. [25], who reported sensitivities between $81 \%$ and $100 \%$ and specificities between $85 \%$ and $100 \%$, which compare well with our results. DOPA-PET and PET/CT were already compared with several other PET tracers (DOTA-TOC/NOC/TATE). Putzer and co-workers evaluated DOTA-TOC vs. DOPA-PET and found sensitivity of $64 \%$, which is somewhat lower than our results and specificity of $100 \%$ for both techniques on a patient-based analysis [27]. However, 68 Ga-DOTA-TOC PET found more lesions (208 malignant lesions vs. 86 lesions) than DOPA-PET.

Ambrosini et al. also found a slight trend of superiority of 68 Ga-DOTA-NOC over DOPA-PET concerning lesion detection [24]. In another study evaluating DOTA-TATE versus DOPA-PET in well-differentiated metastatic neuroendocrine tumours, Haug et al. found sensitivities of $96 \%$ for 68 Ga-DOTA-TATE versus 56\% for DOPA-PET [28]. The reason for the somewhat lower results might be that only well-differentiated neuroendocrine tumours were evaluated.

\section{Incremental clinical value of CT}

To our knowledge, this is the first study to investigate an integrated approach of a combined DOPA-cePET/CT with emphasis on the value of the CT component.

Koopmans et al. showed that a corresponding CT component can add significant information for the diagnosis, leading to increased detection rates in most of the body regions. In our study, adequate multi-phase $\mathrm{CT}$ in combined DOPA-cePET/CT added significant information to the diagnostic sensitivity compared with DOPA-PET but, based on the above mentioned false positive lesions, at the cost of decreased specificity.

Koopmans et al. investigated in another study the usefulness of DOPA-PET and CT in carcinoids vs. its usefulness in islet cell carcinomas [12]. In carcinoid tumour patients, per-patient analysis showed sensitivities for DOPAPET and CT of $96 \%$ each. The per-lesion analysis revealed sensitivities for DOPA PET, DOPA PET/CT and CT alone of $87 \%, 98 \%$ and $63 \%$. While the results for DOPA- PET/CT again compare very well with our results, we found lower values for the DOPA-PET, but higher values for the CT component. The reasons are probably that we investigated an inhomogeneous patient population compared to patients having only carcinoids. The lower results of $\mathrm{CT}$ are explainable by the used one-phase contrast $\mathrm{CT}$, while we used a multi-phase CT. We found one large study that investigated PET in NET vs. adequately performed multiphase CT, but the PET was conducted with DOTA-TOC and not DOPA [29]. This study comprised (like ours) a mixed patient population. This study found somewhat lower values for sensitivity, specificity and accuracy at least for the patient-based analysis. Unlike our study, the CT was conducted separately and was co-registered afterwards to the PET which might explain some of the differences.

Several studies already indicated the therapeutic usefulness of DOPA-PET and PET/CT compared with previous conventional imaging [30-32]. Ambrosini and co-workers e.g. found a significant impact in therapy management induced by DOPA-PET/CT compared with conventional imaging. However, neither contrast-enhanced PET/CT was evaluated nor the diagnostic impact of the CT component itself. Montravers et al. demonstrated that DOPA-PET can have a significant therapeutic impact on patient management but here again, the PET component was evaluated concerning the therapeutic impact.

At first glance, the therapeutic impact of the ceCT component seems rather small in our study because most of the lesions are certainly detected by the DOPA component of PET/CT and ceCT made its major impact in DOPAnegative lesions. There are two aspects to consider: almost every patient who is a candidate for curative or palliative surgery will receive an adequate morphological imaging procedure (most cases: ce-CT, less often MRI) before the surgical procedure. Thus, an integrated "one-stop-shop" imaging approach is certainly desirable for the patients. In these cases, exact anatomical localisation is important, because neuroendocrine tumours of the GI tract in particular can be very small [33]. The second aspect is follow-up in patients after systemic therapy. Our study showed that the CT component in DOPA-negative lesions (after therapy) can add significant diagnostic and therapeutic value to the $\mathrm{PET} / \mathrm{CT}$ procedure.

\section{Limitations/controversies}

Our study has several limitations. Histopathological verification was obtained in a significant number but not in all 
patients after the DOPA-cePET/CT. In several patients no tumour was found and several patients already had an advanced stage with clear clinical signs and were therefore treated systemically without further/additional verification. We did not pre-treat our patients with Carbidopa. Although it has been shown that such pre-treatment can increase the DOPA uptake in tumours/lesions, there is no comparison between pre-treated and non-pre-treated patients available in the current literature [34].

\section{Conclusion}

Combined DOPA-cePET/CT proved to be significantly superior to DOPA-PET and multi-phase CT concerning diagnostic accuracy. While the DOPA-PET showed high specificities, the multi-phase CT component added sensitivity in lesion detection and had therapeutic impact especially in DOP-negative lesions. Overall, combined DOPA-cePET/CT seems to be a robust "one-stop-shop" approach, and has the potential to improve cancer diagnostics in patients with a variety of neuroendocrine tumours.

Acknowledgements This study was partly sponsored by Covidien, Mallinckrodt, Switzerland

\section{References}

1. Lepage C, Bouvier AM, Phelip JM, Hatem C, Vernet C, Faivre J (2004) Incidence and management of malignant digestive endocrine tumours in a well defined French population. Gut 53:549553

2. Modlin IM, Kidd M, Latich I, Zikusoka MN, Shapiro MD (2005) Current status of gastrointestinal carcinoids. Gastroenterology 128:1717-1751

3. Taal BG, Visser O (2004) Epidemiology of neuroendocrine tumours. Neuroendocrinology 80(Suppl 1):3-7. doi:10.1159/ 000080731

4. Yao JC, Hassan M, Phan A et al (2008) One hundred years after "carcinoid": epidemiology of and prognostic factors for neuroendocrine tumors in 35,825 cases in the United States. J Clin Oncol 26:3063-3072. doi:10.1200/JCO.2007.15.4377

5. Gibril F, Reynolds JC, Doppman JL et al (1996) Somatostatin receptor scintigraphy: its sensitivity compared with that of other imaging methods in detecting primary and metastatic gastrinomas. A prospective study. Ann Intern Med 125:26-34

6. Hoegerle S, Altehoefer C, Ghanem N, Brink I, Moser E, Nitzsche E (2001) 18F-DOPA positron emission tomography for tumour detection in patients with medullary thyroid carcinoma and elevated calcitonin levels. Eur J Nucl Med 28:64-71

7. Jager PL, Koopmans KP, de Vries EG (2006) Gastroenteropancreatic neuroendocrine tumours (carcinoid tumours): definition, clinical aspects, diagnosis and therapy. Ned Tijdschr Geneeskd 150:2401-2403, author reply 2403

8. Krausz Y, Keidar Z, Kogan I et al (2003) SPECT/CT hybrid imaging with $111 \mathrm{In}$-pentetreotide in assessment of neuroendocrine tumours. Clin Endocrinol (Oxf) 59:565-573.
9. Lebtahi R, Cadiot G, Mignon M, Le Guludec D (1998) Somatostatin receptor scintigraphy: a first-line imaging modality for gastroenteropancreatic neuroendocrine tumors. Gastroenterology 115:10251027

10. Ambrosini V, Campana D, Bodei L, et al 68 Ga-DOTANOC PET/ CT clinical impact in patients with neuroendocrine tumors. J Nucl Med. doi:10.2967/jnumed.109.071712

11. Koopmans KP, de Vries EG, Kema IP et al (2006) Staging of carcinoid tumours with 18F-DOPA PET: a prospective, diagnostic accuracy study. Lancet Oncol 7:728-734. doi:10.1016/S1470204570801-4

12. Koopmans KP, Neels OC, Kema IP et al (2008) Improved staging of patients with carcinoid and islet cell tumors with $18 \mathrm{~F}$ dihydroxy-phenyl-alanine and 11C-5-hydroxy-tryptophan positron emission tomography. J Clin Oncol 26:1489-1495. doi:10.1200/JCO.2007.15.1126

13. Antoch G, Kanja J, Bauer S et al (2004) Comparison of PET, CT, and dual-modality PET/CT imaging for monitoring of Imatinib (STI571) therapy in patients with gastrointestinal stromal tumors. J Nucl Med 45:357-65

14. Antoch G, Stattaus J, Nemat AT et al (2003) Non-small cell lung cancer: dual-modality PET/CT in preoperative staging. Radiology 229:526-533

15. Beyer T, Townsend DW, Blodgett TM (2002) Dual-modality PET/ CT tomography for clinical oncology. Q J Nucl Med 46:24-34

16. Veit-Haibach P, Kuehle CA, Beyer T et al (2006) Whole-body PET/CT-colonography: diagnostic accuracy of a new staging concept in patients with colorectal cancer. JAMA 296:2590-2600

17. Valk PE, Bailey DL, Townsend DW, Maisey MN (2003) Positron emission tomography: basic science and clinical practice. Springer, London

18. Ahlstrom H, Eriksson B, Bergstrom M, Bjurling P, Langstrom B, Oberg K (1995) Pancreatic neuroendocrine tumors: diagnosis with PET. Radiology 195:333-337

19. Beheshti M, Pocher S, Vali R et al (2009) The value of $18 \mathrm{~F}$ DOPA PET-CT in patients with medullary thyroid carcinoma: comparison with 18F-FDG PET-CT. Eur Radiol 19:1425-1434. doi:10.1007/s00330-008-1280-7

20. Hoegerle S, Altehoefer C, Ghanem N et al (2001) Whole-body $18 \mathrm{~F}$ dopa PET for detection of gastrointestinal carcinoid tumors. Radiology 220:373-380

21. Hoegerle S, Ghanem N, Altehoefer C et al (2003) 18F-DOPA positron emission tomography for the detection of glomus tumours. Eur J Nucl Med Mol Imaging 30:689-694. doi:10.1007/s00259-003-1115-3

22. Koopmans PJ, Barth M, Norris DG Layer-specific BOLD activation in human V1. Hum Brain Mapp. doi:10.1002/ hbm.20936

23. Nanni C, Fanti S, Rubello D (2007) 18F-DOPA PET and PET/CT J Nucl Med 48:1577-1579. doi:10.2967/jnumed.107.041947

24. Ambrosini V, Nanni C, Zompatori $M$, et al Ga-DOTA-NOC $\mathrm{PET} / \mathrm{CT}$ in comparison with $\mathrm{CT}$ for the detection of bone metastasis in patients with neuroendocrine tumours. Eur J Nucl Med Mol Imaging 37:722-727. doi:10.1007/s00259-0091349-9

25. Becherer A, Szabo M, Karanikas G et al (2004) Imaging of advanced neuroendocrine tumors with F-FDOPA PET. J Nucl Med 45:1161-1167

26. Ambrosini V, Tomassetti P, Rubello D et al (2007) Role of 18Fdopa PET/CT imaging in the management of patients with 111Inpentetreotide negative GEP tumours. Nucl Med Commun 28:473477. doi:10.1097/MNM.0b013e328182d606

27. Putzer D, Gabriel M, Kendler D, et al Comparison of Ga-DOTATyr-octreotide and F-fluoro-L-dihydroxyphenylalanine positron emission tomography in neuroendocrine tumor patients. Q J Nucl Med Mol Imaging 54:68-75. 
28. Haug A, Auernhammer CJ, Wangler B et al (2009) Intraindividual comparison of 68 Ga-DOTA-TATE and 18F-DOPA PET in patients with well-differentiated metastatic neuroendocrine tumours. Eur J Nucl Med Mol Imaging 36:765-770. doi:10.1007/s00259-0081030-8

29. Gabriel M, Oberauer A, Dobrozemsky G et al (2009) 68 GaDOTA-Tyr3-octreotide PET for assessing response to somatostatin-receptor-mediated radionuclide therapy. J Nucl Med 50:1427-1434. doi:10.2967/jnumed.108.053421

30. Montravers F, Kerrou K, Nataf V et al (2009) Impact of fluorodihydroxyphenylalanine- $18 \mathrm{~F}$ positron emission tomography on management of adult patients with documented or occult digestive endocrine tumors. J Clin Endocrinol Metab 94:12951301. doi:10.1210/jc.2008-1349
31. Ambrosini V, Campana D, Bodei L et al (2010) 68 Ga-DOTANOC PET/CT clinical impact in patients with neuroendocrine tumors. J Nucl Med 51:669-673. doi:10.2967/jnumed.109.071712

32. Schiesser M, Veit-Haibach P, Muller MK et al (2010) Value of combined $6-[18 \mathrm{~F}]$ fluorodihydroxyphenylalanine PET/CT for imaging of neuroendocrine tumours. Br J Surg 97:691-697. doi:10.1002/bjs.6937

33. Sjoblom SM (1988) Clinical presentation and prognosis of gastrointestinal carcinoid tumours. Scand J Gastroenterol 23:779-787

34. Timmers HJ, Hadi M, Carrasquillo JA et al (2007) The effects of carbidopa on uptake of 6-18F-Fluoro-L-DOPA in PET of pheochromocytoma and extraadrenal abdominal paraganglioma. J Nucl Med 48:1599-1606. doi:10.2967/jnumed.107.042721 